\title{
MPLS BACKBONES AND QOS ENABLED NETWORKS INTEROPERATION
}

\author{
Fernando Manchini Serenato ${ }^{1}$, Juergen Rochol ${ }^{2}$ \\ 1 Pontifícia Universidade Católica do Paraná (PUCPR) \\ Av. Maringá, 78 - 86060-000 - Londrina - PR - Brasil \\ serenato@rla01.pucpr.br \\ ${ }^{2}$ Instituto de Informática - Universidade Federal do Rio Grande do Sul (UFRGS) \\ Caixa Postal 15.064 - 91.501-970 - Porto Alegre - RS - Brasil \\ juergeneinf.ufrgs.br
}

\begin{abstract}
It is believed that in the future the telecommunication and data networks will be integrated into one single network, based on IP switching. One of the technologies that certainly will be adopted in the core of this new network is MPLS. Together with MPLS, other QoS architectures, such as IntServ and DiffServ will be used. As a result of this, a framework that allows the interoperability of the different existing architectures is needed. In this paper, an integration model based on MPLS and DiffServ is presented. The model was tested in the ns 2 simulator and the results certify its applicability as the presented model showed better performance in all analyzed QoS parameters: delay, jitter, packet loss and bandwidth.
\end{abstract}

Keywords: Quality of Service, MPLS, Interoperation

\section{Introduction}

Currently, most of the Internet infrastructure supports only the best effort service. Packets are processed in the fastest possible way, however, there are no guarantees about

Permission to make digital or hard copies of all or part of this work for personal or classroom use is granted without fee provided that copies are not made or distributed for profit or commercial advantage, and that copies bear this notice and the full citation on the first page. To copy otherwise, to republish, to post on servers or to redistribute to lists, requires prior specific permission and/or a fee.

LANC'03, October 3-5, 2003, La Paz, Bolivia

(C2003 ACM 1-58113-789-3/03/0010 Order n. 103035 ...\$5.00 delay and not even if it will be delivered. Previously, this did not represent a problem, but with the recent popularization of the Internet and the consequent traffic growth, demands appeared for services with guaranteed quality.

Furthermore, there exists the problem of many Internet links already presenting signals of chronic congestion. Thus, to solve these problems, two alternatives are proposed: over provisioning the network, using technologies such as DWDM (Dense Wave-lenght Division Multiplex) and Gigabit Ethernet, or else, use packet switching technologies and Traffic Engineering to minimize the congestion and the delay of the network.

The first solution is not cheap and will not satisfactorily use the resources provided by the network. Also, whenever exists surplus bandwidth, new applications will be created to use it. The second alternative, based on a IP (Internet Protocol) framework, intends to transform the Internet into a network with services integration and flexibility to support the new appearing applications. All this with different levels of Quality of Service (QoS) provision.

The IETF (Internet Engineering Task Force) considered several architectures for QoS provisioning, amongst them are: the Integrated Services (Intserv) architecture [Braden 1994], using RSVP [Braden 1997] as the signaling protocol, the Differentiated 
Services (Diffserv) architecture [Blake 1998], [Nichols 1998], the Constraint Based Routing concept [Crawley 1998], [Jamoussi 2002], and more recently MPLS (MultiProtocol Label Switching) [Rosen 2001], [Magalhães 2001].

The Intserv architecture [Braden 1994] is based on network resource reservation. Before initiating a transmission paths are configured, and the necessary resources reserved. These reservations can be performed by RSVP [Braden 1997], a path signaling and configuration protocol.

In the Diffserv architecture [Blake 1998], [Nichols 1998], the packets are marked with different values, creating several classes of packets. Packets belonging to different classes receive different levels of service.

Constraint Based Routing (CBR) [Crawley 1998], [Jamoussi 2002] is a more sophisticated way of route selection, which consists of finding routes in the network that take into consideration other metrics, such as available bandwidth or maximum delay, and not only shortest path, as the current IGPs (Interior Gateway Protocols) do.

MPLS [Rosen 2001], [Magalhães 2001] provides IP packet switching based on a label, which the packets receive when they enter a MPLS domain. The subsequent classification and commuting are based only on the label, accelerating the process. MPLS also allows sophisticated routing and path setup capabilities in the form of explicit routed LSPs (Label Switched Paths). This is the foundation of traffic engineering using MPLS [Swallow 1999]. Paths can be setup depending on the type of traffic, or based in the current network load. This allows an equal division of the traffic through the links, optimizing its use and diminishing the congestion possibilities.

Taking into consideration the advantages presented by MPLS, it will be the technology of choice for the Internet Service Suppliers (ISPs) in the near future. However, the migration to MPLS is not trivial [Xiao 2000]. Because of this, it is given credit that this migration, in a first moment, will only be made by the companies who will benefit more of it: the ISPs. For the other companies, solutions that present satisfactory levels of QoS, without a great expense or reorganization of the network will be enough. With this scenario appears the need of a framework which will allow the interoperation of the different QoS architectures used in the companies, such as Intserv or Diffserv, and the ISPs technologies, such as MPLS. Presenting that framework is the main objective of this paper.

The organization of the rest of this paper is as follows. In section 2 the reasons for the current QoS architectures integration are presented and also, several other integration models are quickly reviewed and compared. The proposed model is presented in section 3. The results of a simulation of the proposed model in the $n s 2$ simulator are presented in section 4. The paper is summarized in section 5 .

\section{Why integrate different QoS architectures?}

Although supplying a high level of resource reservation guarantee, Intserv has serious scalability and complexity problems. Diffserv, in turn, is simple and scalable, but presents deficiencies in treating different flows in the same manner (aggregates). Another problem is that Diffserv was planned for IP, but it would be interesting to apply it directly in data link layer technologies such as ATM and Frame Relay.

TABLE 1 - Comparison of the integration models 


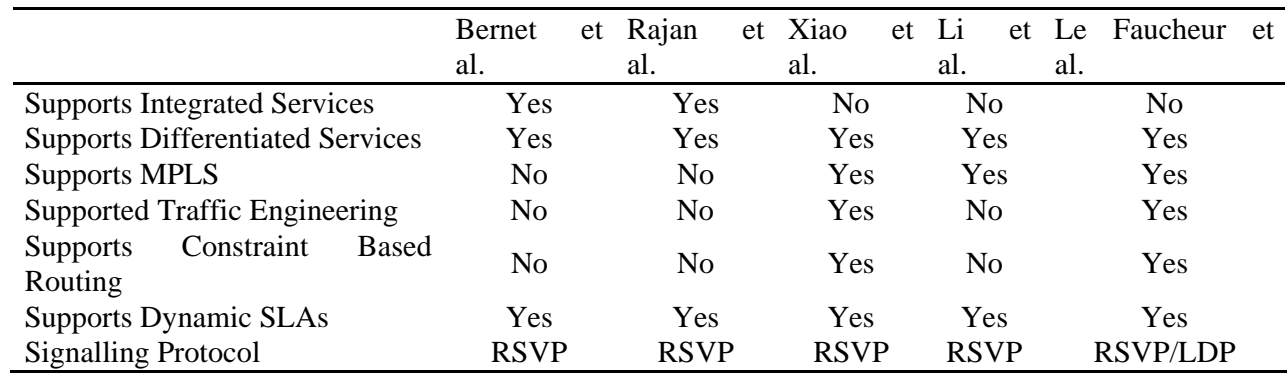

Both Intserv and Diffserv use network layer IP routing. However, this process is slower than data link layer commutation. Moreover, the routing process is normally based on SPF (Shortest Path First) which does not use efficiently the network infrastructure causing bottlenecks. These problems can be solved by using Traffic Engineering and MPLS.

The observation that none of the architectures alone takes care of all the endto-end QoS requirements, and the fact that the Internet is a heterogeneous network, where there will always exist the need of different technologies interoperation, has lead to the proposal of several models that combine these architectures. These are seen as complementary in the end-to-end service provisioning. In this way, these models try to join all the architectures advantages into only one implementation, such as in [Le Faucheur 2002], [Bernet 2000], [Xiao 1999], [Rajan 1999] and [Li 1998].

[Bernet 2000] considers a model that combines the Intserv / RSVP architecture with Diffserv. It uses Diffserv in the core networks, called transit networks, and Intserv in the adjacent customer's networks.

Some theoretical proposals, as [Xiao 1999] and [Rajan 1999], have been presented suggesting improvements or variations in the model presented by [Bernet 2000]. [Rajan 1999] specifies access regulation to the resources and services of the network being based on administrative criteria, and [Xiao 1999] compares different integration models using IntServ, Diffserv and MPLS.

More recently, [Le Faucheur 2002] considered an architecture for MPLS and Diffserv integration where Behavior Aggregates (BAs) are mapped in LSPs. A set of BAs can be mapped in one LSP (ELSP: EXP-Inferred-PSC LSP), or a unique BA can be mapped in a LSP (L-LSP: LabelOnly-Inferred-PSC LSP). In this way, it is possible to satisfy the needs of service differentiation, traffic engineering and flow protection for each network.

\subsection{Comparison of the integration models} The presented integration models have sufficiently distinct characteristics ones of the others. Each one supports a different set of QoS supplying architectures and uses different signaling protocols. A summary of these characteristics can be found in Table 1 .

The model proposed in this paper intends to support all these criteria, in a flexible and scalable form, without sacrificing the general performance of the network. This model will be presented in the next item.

\section{Proposed Model}

The model proposed in this paper indicates simple procedures so that a MPLS domain supplies end-to-end service guarantees to the neighboring customer's networks. These networks could be using Diffserv, Intserv, MPLS, or even no QoS supplying architecture. The proposed model is an extension to the [Le Faucheur 2002] model, 


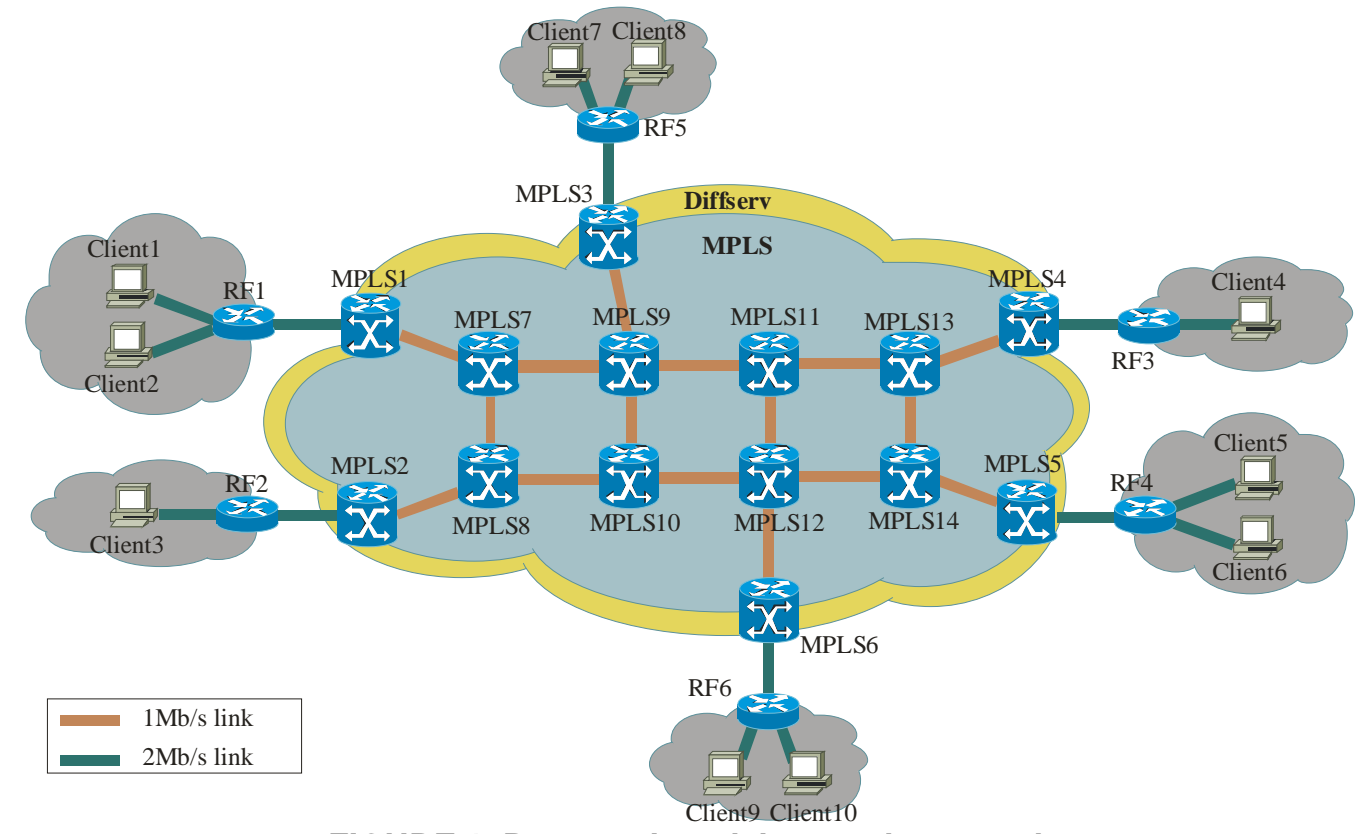

FIGURE 1. Proposed model example network

upgrading it to support Intserv flows on a network using MPLS and Diffserv.

Since MPLS is a technology destined to the core of today's networks, one of its main characteristics is scalability, which is reached through flow aggregation with endto-end service guarantees, and without the need of individual flow control in each segment of its path. Thus, the Diffserv architecture become very interesting for QoS supplying inside of MPLS domains as its services are based on a per-hop model and resources, as buffer space and bandwidth, which are pre-allocated in the LSRs for each service. Functions as classification, marking and policing are performed only in the edges of the network while the core LSRs only needs classifiers, keeping its simplicity and scalability.

Figure 1 shows an example network that uses the proposed model: in the network core there are only MPLS LSRs, which will also play the role of Diffserv core routers as in [Le Faucheur 2002]. In the network edges are the LSRs which will also behave as Diffserv edge routers, and moreover, will also do the customer network service mapping into the proposed model services. Linked to the edge LSRs there are several customer's networks. Beyond the inherent advantages of using MPLS and Diffserv together, the following reasons had led to the choice of Le Faucheur's model for the network core:

- BAs to LSPs mapping flexibility;

- Supports current and future PHBs;

- Flexibility for the ISPs to choose how the service classes will be routed inside of its domain;

- Use of MPLS offers greater protection and restoration capacity when the topology changes;

- Flexibility in the choice of the protection and restoration levels;

- Conservative use of the available label space;

- When possible, it optimizes the amount of setup and tear-down messages for LSPs;

- Supports IPv4 and IPv6.

The [Le Faucheur 2002] model does not support Intserv. Since the main objective 
TABLE 2. Service Mapping

\begin{tabular}{lllc}
\hline Integrated services & Differentiated services & Proposed model & EXP Field \\
\hline Guaranteed (GS) & Expedited Forwarding (EF) & Gold class & 111 \\
Controlled load (CL) & Assured Forwarding (AF) AFx1 & Silver class & $110 / 011$ \\
& AFx2 AFx3 & Bronze class & $101 / 010$ \\
& & & $100 / 001$ \\
Best effort & Best effort & Best effort & 000 \\
\hline
\end{tabular}

of the proposed model is the interoperation of the main existing QoS supplying architectures, it is necessary to add Intserv capabilities to Le Faucheur's [Le Faucheur 2002] mapping procedure. To do this mapping, the procedures considered in [Bernet 2000] for operation with resources dynamically provisioned by RSVP will be used. The main advantages of this operation mode are:

- It allows the negotiation of dynamic SLAs; Changes in the available bandwidth of the network core can be signaled for the customer's network through RSVP;

- It is possible to change the network core provisioning through RSVP and through the procedures presented in [Ash 2002].

The use of this set of procedures leads to a flexible, yet scalable model, which can be used by the service suppliers to support several customers' networks.

\subsection{Supported services}

To support service differentiation, the proposed model specifies the following traffic classes:

- Gold class: a low delay, low jitter and few losses service, for delay sensitive flows. In this class, packets that exceed the SLA configured maximum transmission rate are dropped;
- Silver and Bronze classes: destined for throughput sensitive flows. Packets int the silver class experience lighter load (and thus have a greater probability for timely forwarding). Furthermore, each class uses two thresholds for packet drop. Packages that exceed the SLA configured maximum transmission rate can be discarded or marked with higher drop precedence;

- Best effort class: no guarantees service.

The ingress LSRs are responsible for the mapping demonstrated in Table 2, based on the SLA established with the customer network. Two distinct situations can be considered: first, the customer's network packets already come with a defined type of service, and it is enough to the ingress LSR to make the mapping. Second, the packages come without indication of type of service. In this case, the ingress LSR must make a multi-field (MF) classification to select the arriving packets new service class.

\subsection{Scheduling and conformation}

ISPs can adopt several scheduling and packet drop disciplines. An example is shown in Figure 2, and consists of 4 queues, one for each service class, with a priority based (PQ) scheduler serving the queues. The Gold queue has the highest priority and the Best effort queue has the lowest priority. 


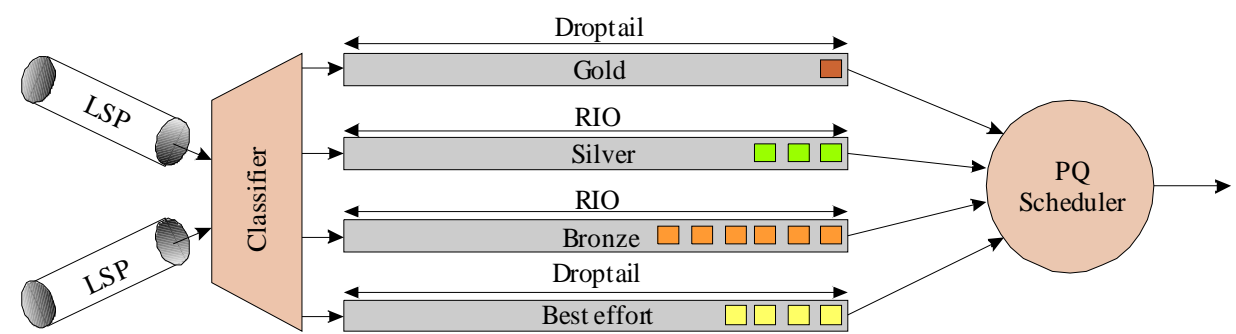

Figure 2. Scheduling in the MPLS network core

Gold and Bests effort class queues uses Droptail queue management. Droptail queues implements FIFO (first in - first out) scheduling and drop-on-overflow buffer management.

Silver and Bronze class queues uses RIO (Random Early Detection with in and out) queue management. RIO is a more advanced RED (Random Early Detection) scheme, it basically maintains two RED algorithms, one for in packets and one for out packets. There are two thresholds for each queue. When the queue size is below the first threshold, no packets are dropped. When the queue size is between the two thresholds, only out packets are randomly dropped. When the queue size exceeds the second threshold, indicating possible network congestion, both in and out packets are randomly dropped, but out packets are dropped more aggressively.

Traffic shaping is done by the ingress LSRs through the use of Token Buckets. The Token Buckets are configured using CIR (Committed Information Rate) and CBS (Committed Burst Size) values. For the Gold and Best effort service classes the CBS is equal to zero, and therefore no traffic excess is allowed.

\subsection{Signaling and service mapping}

A customer who desires to use the MPLS domain (ISP) services must establish a SLA with it. The SLA can be static or dynamic. In the case of dynamic SLAs establishment some signaling protocol, such as RSVP, is necessary. To minimize the establishment time of the LSPs, the ISP network administration can statically allocate the resources and configure the LSPs based on the customer's necessities.

The signalling between the customer networks and the MPLS domain can be done by RSVP, allowing the establishment of PHB $\rightarrow$ FEC (Forwarding Equivalence Class) relationships, and perhaps, the dynamic establishment of a new LSP (Dynamic SLAs). Inside the MPLS domain the signalling protocol is LDP [Andersson 2001], and it's extension for Constraint Based Routing, CR-LDP [Jamoussi 2002]. The ingress LSRs will also do admission control, based on the information provided by the MPLS domain.

\subsection{Operation}

As well as in Diffserv, the core and edge LSRs presents different functions and responsibilities, as seen in Figure 3. In common they have:

- Constraint based routing;

- CR-LSPs establishment;

- Admission control;

- Scheduling mechanisms. 


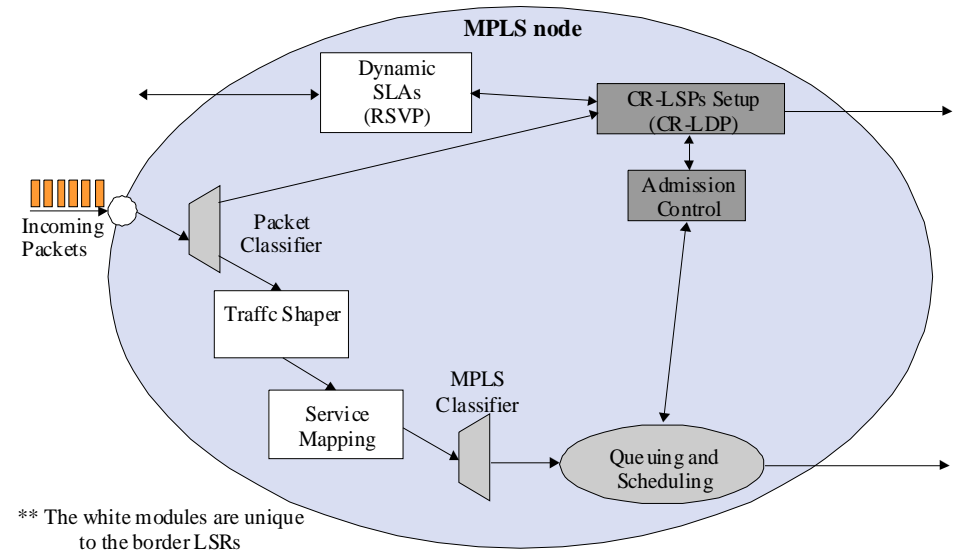

FIGURE 3. MPLS node internal structure

Additionally, the ingress LSRs are responsible for:

- Traffic classification;

- Traffic policing;

- Traffic shaping;

- Customer network to proposed model service mapping.

In the MPLS domain core there will be two types of LSP: with the E-LSP it will be possible that BAs (Behavior Aggregates) share the configured bandwidth. To differentiate each one of BAs, the EXP field of the Shim Header will be used. It will hold the DSCP and drop precedence. If the network core is based on a data-link technology that do not uses the Shim Header, other mappings using the data-link technology characteristics is used. This type of LSP will allow a bigger aggregation of flows, resulting in a lesser number of LSPs configured, and consequently, facilitating the Traffic Engineering.

A shim header is a special header placed between layer two and layer 3 of the OSI model. The shim header contains the label used to forward the MPLS packets.

The second type of LSP, the L-LSP, carry only one aggregate, whose QoS needs are strict. In this case the packets' DSCP is inferred through it own label. Separating it in an exclusive LSP, its isolation is increased, and a sufficiently strict QoS can be guaranteed. The division of the LSPs in two types also facilitates the Traffic Engineering, and also allows the network administrator to know accurately what type of aggregate is flowing through which link of the network in any instant of time.

The packet routing inside an MPLS domain will be made using the packets' MPLS label and the forwarding treatment, or PSC (PHB Scheduling Class), applied by each LSR to the packets will be based on the DSCP and the drop precedence, both coded in the EXP field (E-LSPs) or in the label (LLSPs) of each packet.

\subsection{Service allocation in the customers' networks}

For the services allocation inside the customers' networks two alternatives are proposed in [Xiao 1999]:

- Each computer decides alone which services it will use;

- A BB (Bandwidth Broker) decides which services each machine will be able to use.

Thus before a computer starts the transmission it can decide alone which service to use or it can query the BB. The computers also can, or not, use some packet marking mechanism. In this way, the sending computer can mark the packets, or send them without any marking leaving this service for the border routers. In the last case 
the border routers will have that to be automatically or manually configured. If SLAs are dynamically negotiated between the customers and the ISPs, the computers, or the BB, will need a signaling protocol to negotiate ISP resources under demand. One of the protocols which could be used for this is RSVP.

\subsection{Allocation of services in the ISPs' network}

For the resource allocation inside the ISPs' networks, the SLAs must be examined and after that the edge LSRs can be configured, ensuring that they can handle the incoming traffic. For static SLAs the routers can be configured manually, statically allocating resorcer for each customer. For dynamic SLAs the resource allocation depends on the signaling method. If the computers, or the $\mathrm{BB}$, from the customer networks use RSVP for the resource requestion, the admission control can be made in a distributed way by the edge LSRs. If the request is accepted, the edge LSRs are configured with the corresponding classification, policing and shaping rules. The ISP's core LSRs does not take part in the signaling to prevent scalability problems.

\section{Simulation and Results}

To validate the proposed model the ns2 simulator (Network Simulator 2) [The network simulator ns-2 2002] was used. ns2 is a event driven simulator, developed at University of California.

To simulate MPLS and Diffserv networks the [Ahn 2001] and [Pieda 2000] extensions are being used. [Ahn 2001] implements MPLS packet switching and of the LDP/CR-LDP message handling. [Pieda 2000] implements the AF (Assured Forwarding) and EF (Expedited Forwarding) services of Diffserv. To correctly run the simulations some modifications, dealing with flow and queue limitations, in the ns2 sources had been necessary.

\subsection{Simulation description}

The topology used in the tests is equal to the one shown in Figure 1, and consists of the following: the ISP network core is composed of 8 MPLS LSRs (MPLS7 until MPLS14) connected through $1 \mathrm{Mb} / \mathrm{s}$ links. These links have deliberately low bandwidth to facilitate its congestion. In the core access points there are 6 edge LSRs (MPLS1 until MPLS6), which make the traffic policing and shaping. The edge LSRs are connected to the core LSRs through $1 \mathrm{Mb} / \mathrm{s}$ links also, for the same previously cited reason.

As a result, one packet passing through the edge LSRs, is policed and marked with a DSCP, classified in a FEC and switched, to the network core. In the tests, only LSPs with the same aggregate priority had been used. Shaping, queuing and scheduling are made as presented in section 4.2. The label distribution is done through LDP in ordered, under demand mode.

Connected to the ISP there are 6 customer networks, with several types of traffic sources, such as CBR, FTP and HTTP. The links which connect the customer networks to the border routers (RF) and these to the MPLS domain edge LSRs have a 2Mbp/s bandwidth. Its bandwidth is bigger than the ISP links so that none of them saturate. The customers considered use the best effort, $\mathrm{AF}$ and $\mathrm{EF}$ services of Diffserv. For the time being, there are no customers using Intserv.

This topology does not aim to simulate an already existing network, but to assist in the analysis of the obtained results. The links' bandwidth is deliberately low to facilitate its congestion and the way they are connected allow the analysis of the data in pre-defined points of the topology.

\subsection{Flows}

Several different flows were simulated. The modeling of the traffic sources is detailed in Table 3: 
TABLE 3. Traffic sources

\begin{tabular}{|c|c|c|c|c|c|}
\hline $\begin{array}{l}\text { Traffic } \\
\text { type }\end{array}$ & Protocol & Packet size & $\begin{array}{l}\text { Interval between } \\
\text { connections }\end{array}$ & Connection duration & $\begin{array}{l}\text { Amount of } \\
\text { flows }\end{array}$ \\
\hline Voice & UDP & 66 bytes & $\begin{array}{l}\text { Exponential } \\
\text { distribution with } \\
\text { average value of } 20 \mathrm{~ms}\end{array}$ & $\begin{array}{l}\text { Exponential distribution } \\
\text { with average value of } \\
180 \mathrm{~ms}\end{array}$ & 2 \\
\hline FTP & TCP & 1500 bytes & -- & -- & 2 \\
\hline HTTP & TCP & $\begin{array}{l}1500 \text { bytes } \\
\max .\end{array}$ & $\begin{array}{l}\text { Uniform distribution } \\
\text { with average value of } \\
0,2 \text { second }\end{array}$ & -- & 3 \\
\hline
\end{tabular}

The voice flows had been shaped so that they simulated VoIP (Voice over IP) flows using CODEC G.729A for compression. Each one of the flows is an aggregate containing 7 simultaneous conversations, with each packet carrying the equivalent to $30 \mathrm{~ms}$ of the conversation. The FTP and HTTP flows packet size is 1500 bytes because of TCP's improved operation with bigger sized packets. The HTTP flows page size was based on the average size of randomly chosen Internet pages.

The simulated flows and its service classes can be seen in Table 4 . The voice flows have a greater priority than the other flows; consequently they are mapped in Gold class. Both FTP flows are mapped in Silver class, therefore it is desired that they have a superior QoS at the same time they can send some traffic bursts. HTTP flows are of lesser priority, for this reason they are mapped in Bronze and Best effort classes. Each service class has also a limit on maximum bandwidth.

For silver and bronze class flows, the ingress LSRs marks all excess traffic with higher drop precedence. This way, in case of congestion, the excess packets will be dropped first. The main objective of the simulation is to show that the MPLS infrastructure is improved in terms of flow provisioning (bandwidth) and separation (to jitter) when it is used jointly with Diffserv mechanisms.

\subsection{Requirements and Results}

For comparison, two simulations had been executed, one with MPLS only and another with the proposed model (Diffserv and MPLS). Each of the simulations was executed 10 times and the presented values (tables and charts included) are the average results of the 10 repetitions. In some cases the standard deviation is also presented.

Table 5 shows a summary of the results. The first section of the table shows the general results without making any flow distinction. It is possible to perceive that the sent and discarded packet average in the two models is practically equivalent. However the predictability of the MPLS model is minor, as demonstrated by its higher standard deviation. The second section of the table shows diverse QoS parameters for each flow in both models.

\section{Delay}

Delay is an extremely important QoS metric for VoIP flows. Analyzing Table 5 it is possible to perceive that flow VOZ1 in the MPLS model suffered an average delay of $206 \mathrm{~ms}$; this is $44 \mathrm{~ms}$ larger than the maximum value specified in the ITU-T G.114 recommendation. Flow VOZ2, in turn, suffered an acceptable delay of $34 \mathrm{~ms}$. Because of these results the MPLS model did not ensure the delay requirements of the voice flows. In the MPLS+Diffserv model, both flows, VOZ1 and VOZ2, were inside the tolerable limit, with average delays of $55 \mathrm{~ms}$ and $28 \mathrm{~ms}$ respectively.

\section{Packet loss}

Packet loss should be always avoided, 
TABLE 4. Simulated flows (Transmission rates in Kbits/s)

\begin{tabular}{llllllll}
\hline Flow & Type & Origin & Destination & TX rate & CIR & CBS & Class \\
\hline VOZ1 & Exponential/UDP & Client2 & Client6 & $\sim 132 \mathrm{~Kb} / \mathrm{s}$ & $150 \mathrm{~Kb} / \mathrm{s}$ & $0 \mathrm{~Kb}$ & Gold \\
VOZ2 & Exponential/UDP & Client6 & Client2 & $\sim 132 \mathrm{~Kb} / \mathrm{s}$ & $150 \mathrm{~Kb} / \mathrm{s}$ & $0 \mathrm{~Kb}$ & Gold \\
FTP1 & FTP/TCP & Client3 & Client4 & -- & $400 \mathrm{~Kb} / \mathrm{s}$ & $1 \mathrm{~Kb}$ & Silver \\
FTP2 & FTP/TCP & Client7 & Client5 & -- & $400 \mathrm{~Kb} / \mathrm{s}$ & $1 \mathrm{~Kb}$ & Silver \\
HTTP1 & HTTP/TCP & Client1 & Client8 & -- & $100 \mathrm{~Kb} / \mathrm{s}$ & $0 \mathrm{~Kb}$ & BE \\
HTTP2 & HTTP/TCP & Client1 & Client9 & -- & $350 \mathrm{~Kb} / \mathrm{s}$ & $1 \mathrm{~Kb}$ & Bronze \\
HTTP3 & HTTP/TCP & Client1 & Client10 & -- & $350 \mathrm{~Kb} / \mathrm{s}$ & $1 \mathrm{~Kb}$ & Bronze \\
\hline
\end{tabular}

independently of the type of flow. However, both voice flows, had suffered packet drops in the MPLS model. Moreover, flows FTP2, HTTP2 and HTTP3 had also suffered packet drops. It must be remembered that flows which use TCP as transport protocol uses its flow control mechanisms to react to the drops, thus lowering its transmission rate. Since the VoIP and FTP flows should be prioritized, the MPLS model has not corresponded to the expectations. In the MPLS + Diffserv model this problem does not occur. Moreover, the total amount of voice and FTP packets sent, is bigger. The only flow that has dropped packets is HTTP1 which is the lesser priority flow (Best Effort).

\section{Jitter}

The jitter values, as well as delay, are very important for the voice flows. Very high jitter values leads to unintelligible conversation. Analyzing Table 5 the average jitter values of both voice flows and also the interval between packet transmissions can be compared. Both models satisfactorily ensure the jitter requirements of the voice flows, with a small advantage, also in standard deviation, for the MPLS + Diffserv model. This advantage comes from using a priority based scheduler.

\section{Bandwidth}

The bandwidth available for each flow is the most important QoS metric for elastic applications such as FTP and HTTP. Observing the results in Table 5 it is possible to confirm that for all flows, with exception of HTTP1, the bandwidth increases when the models are swapped. The HTTP3 bandwidth diminishes because it is the lesser priority flow. The best example is FTP1 which has a rough increase of $13 \%$ in its throughput. Since the sending rate of the VoIP flows slightly varies, the throughput of FTP flows represents the performance profit gotten by the average priority traffic when the HTTP traffic is shaped through the Diffserv mechanisms. This shows that the MPLS+Diffserv model also ensure the QoS of the bandwidth dependent flows.

The following charts allow a more detailed analysis. The lack of traffic separation and prioritizing in the MPLS model is evident when VOZ1 jitter is analyzed in Figure 4. Its variation in the MPLS+Diffserv is well smaller. FTP1 is mapped into Silver class. Thus one expects that it gets a better QoS then Bronze and Best effort classes. This will be achieved through its prioritizing. Figure 5 shows that this objective is reached in the MPLS+Diffserv model. The bandwidth received by FTP1 is nearly constant, around $400 \mathrm{~Kb} / \mathrm{s}$, which is the maximum configured for Silver class. HTTP1 is the flow with lesser priority, therefore it belongs to the Best effort class. Its received bandwidth is shown in Figure 6. In the MPLS model, for its lack of prioritizing and shaping mechanisms, it troubles the transmission of other flows. In the MPLS+Diffserv model its bandwidth is limited to $100 \mathrm{~Kb} / \mathrm{s}$. 
TABLE 5. Simulation results

\begin{tabular}{|c|c|c|c|c|}
\hline & MPLS (Average) & Standard deviation & MPLS+Diffserv (Avg.) & Standard deviation \\
\hline Total sent packets & $9.146,4$ & 50 & $9.244,4$ & 11,5 \\
\hline Discarded packets & 19 & 3,2 & 6,2 & 1,3 \\
\hline \multicolumn{5}{|l|}{ Lost packets } \\
\hline VOZ1 & 8,6 & 6,6 & 0 & 0 \\
\hline VOZ2 & 4,2 & 9,4 & 0 & 0 \\
\hline FTP2 & 2,4 & 1,1 & 0 & 0 \\
\hline HTTP1 & 0 & 0 & 6,2 & 1,3 \\
\hline HTTP2 & 0,4 & 0,9 & 0 & 0 \\
\hline HTTP3 & 0,2 & 0,4 & 0 & 0 \\
\hline \multicolumn{5}{|l|}{ Average delay } \\
\hline VOZ1 & $0,206 \mathrm{~s}$ & $0,009 \mathrm{~s}$ & $0,055 \mathrm{~s}$ & $0,0003 \mathrm{~s}$ \\
\hline VOZ2 & $0,034 \mathrm{~s}$ & $0,0004 \mathrm{~s}$ & $0,028 \mathrm{~s}$ & $0,000005 \mathrm{~s}$ \\
\hline \multicolumn{5}{|l|}{ Bandwidth } \\
\hline VOZ1 & $133,52 \mathrm{Kbit} / \mathrm{s}$ & -- & $139,21 \mathrm{Kbit} / \mathrm{s}$ & -- \\
\hline VOZ2 & $131,39 \mathrm{Kbit} / \mathrm{s}$ & -- & $141,31 \mathrm{Kbit} / \mathrm{s}$ & -- \\
\hline FTP1 & $256,98 \mathrm{Kbit} / \mathrm{s}$ & -- & $290,51 \mathrm{Kbit} / \mathrm{s}$ & -- \\
\hline FTP2 & $215,81 \mathrm{Kbit} / \mathrm{s}$ & -- & $229,37 \mathrm{Kbit} / \mathrm{s}$ & -- \\
\hline HTTP1 & $47,65 \mathrm{Kbit} / \mathrm{s}$ & -- & $24,74 \mathrm{Kbit} / \mathrm{s}$ & -- \\
\hline HTTP2 & 40,48Kbit/s & -- & $43,06 \mathrm{Kbit} / \mathrm{s}$ & -- \\
\hline HTTP3 & $38,53 \mathrm{Kbit} / \mathrm{s}$ & -- & $42,81 \mathrm{Kbit} / \mathrm{s}$ & -- \\
\hline \multicolumn{5}{|c|}{ Interval between packet transmissions } \\
\hline VOZ1 & $0,03544 \mathrm{~s}$ & $0,010 \mathrm{~s}$ & $0,03561 \mathrm{~s}$ & $0,012 \mathrm{~s}$ \\
\hline VOZ2 & $0,03598 \mathrm{~s}$ & $0,013 \mathrm{~s}$ & $0,03564 \mathrm{~s}$ & $0,012 \mathrm{~s}$ \\
\hline \multicolumn{5}{|l|}{ Jitter } \\
\hline VOZ1 & $0,03571 \mathrm{~s}$ & $0,006 \mathrm{~s}$ & $0,03567 \mathrm{~s}$ & $0,005 \mathrm{~s}$ \\
\hline VOZ2 & $0,03615 \mathrm{~s}$ & $0,015 \mathrm{~s}$ & $0,03572 \mathrm{~s}$ & $0,007 \mathrm{~s}$ \\
\hline
\end{tabular}

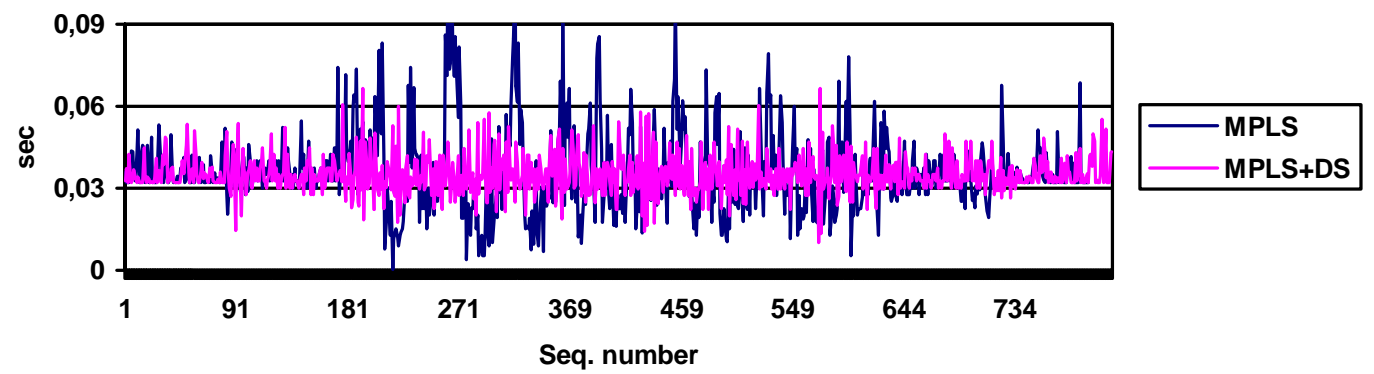

FIGURE 4. Jitter presented for the Voz1 flow

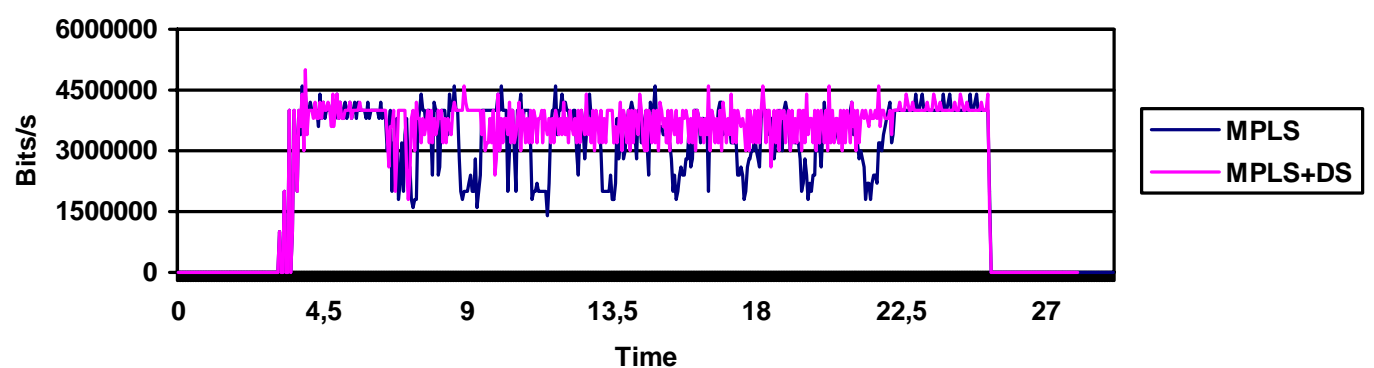

FIGURE 5. Bandwidth received for flow FTP1 


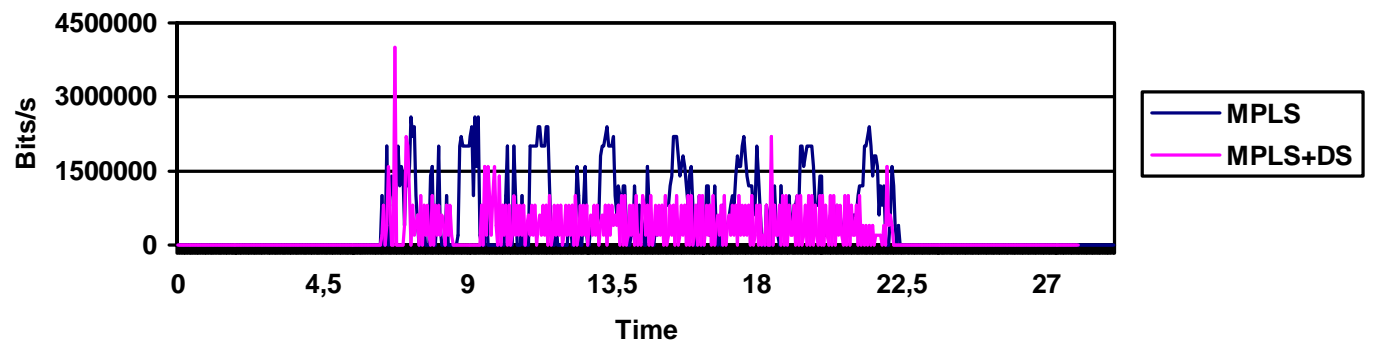

FIGURE 6.Bandwidth received for flow HTTP1

\section{Conclusion}

IP networks, and notably the Internet, are quickly evolving into a commercial infrastructure, crucial to diverse companies and users. Thanks to this transformation, several demands also appeared claiming for an improvement in the quality of the offered services. Instead of only one type of service (Best effort), the services will be offered through service classes with different quality of service parameters. The price to be paid is proportional to the desired quality of service. The companies eager that its customers get fast access to its products, will pay the price to offer faster, efficient and trustworthy services, being able to contract only one, or several, service class, and using them for diverse dealings, beyond the data exchange with the customers.

Several architectures had been proposed by the IETF to provide Quality of Service (QoS). Each one of these architectures presents advantages and disadvantages. The observation that the Internet is a heterogeneous network, where there will always exist the need of different technologies interoperation, has lead to the proposal of several models that combine these architectures.

This paper presents a QoS architectures integration model that allows established Diffserv, Intserv, MPLS or even no-QoS aware customer networks to use the services offered by an MPLS+Diffserv backbone. Thus, in the backbone, Diffserv's scalability and simplicity is paired with MPLS' fast switching. Since it is not necessary to keep state information in each core node, scalability is increased. The proposed model considers 4 service classes: Gold, Silver, Bronze and Best effort. The flows aggregation/separation in LSPs facilitates Traffic Engineering, allowing the network supervisor to know accurately what kind of flow is going through its network infrastructure. Additionally, taking into consideration the QoS and Traffic Engineering restrictions, CBR could be used in order to find the best routes in the network core. This paper also shows how the proposed model can interoperate with the customer networks. Consequently, a complete end-to-end QoS architecture has been reached:

- Traffic handling mechanisms: based in the DSCP, the drop precedence and MPLS label;

- Configuration mechanism: RSVP externally (dynamic SLAs) and LDP/CR-LDP internally;

- Provisioning: admission control in the edge LSRs and PSCs in the core LSRs.

To evaluate the functionality of the proposed model, the ns 2 network simulator was used. To validate the simulation the 
insight of specialists were used, as there is not any complete laboratory for the tests, where it would be possible to evaluate the behavior of the proposed model. Several situations had been simulated to test the behavior of the proposed model in different critical circumstances. The used configurations reflect, to a large extent, possible configurations in the existing routers, and no inexistent resources in the current firmware implementations were used.

The obtained results suggest the applicability of the proposal. The MPLS+Diffserv demonstrated better performance in all the analyzed QoS parameters: delay, jitter, packet loss and bandwidth.

Delay and jitter are very important for real time flows such as VoIP. Bandwidth and packet loss are important for the elastic applications flows, such as FTP and HTTP. In the simulations, with real time and elastic flows, the QoS parameters had improved for both flow types in the transposition from the MPLS model to the MPLS+Diffserv model, suggesting the validity of the model. In addition, the network performance, in general terms, was also improved.

\section{References}

Ahn, G.; Chun W. (2001) Design and Implementation of MPLS Network Simulator supporting LDP and CR-LDP. In: IEEE International Conference on Information Networking, ICOIN, 15., 2001, Oita-Japan. Proceedings... New York: IEEE Press, 2001.

Andersson, L. et al. (2001) LDP Specification. [S.1.]: IETF, Jan. 2001. (RFC 3036).

Ash, J. et al. (2002) LSP Modification using CR-LDP. [S.1.]: IETF, Jan. 2002. (RFC 3214).

Bernet, Y. et al (2000). A Framework for Integrated Services Operation over
Diffserv Networks. [S.1.]: IETF, Nov. 2000. (RFC 2998).

Blake, S. et al. (1998) An Architecture for Differentiated Services. [S.1.]: IETF, Dec. 1998. (RFC 2475).

Braden, R. et al. (1994) Integrated Services in the Internet Architecture: an Overview. [S.1.]: IETF, June 1994. (RFC 1633).

Braden, R. et al. (1997) Resource ReSerVation Protocol (RSVP) - Version 1 Functional Specification. [S.1.]: IETF, Sept. 1997. (RFC 2205).

Crawley, E. et al. (1998) A Framework for QoS-based Routing in the Internet. [S.1.]: IETF, Aug. 1998. (RFC 2386).

Jamoussi B. (Ed.) (2002). Constraint-Based LSP Setup using LDP. [S.1.]: IETF, Jan. 2002. (RFC 3212).

Le Faucheur, F. et al (2002). MPLS Support of Differentiated Services. [S.1.]: IETF, May. 2002. (RFC 3270).

Magalhães, M.; Cardozo, E. (2001) Introdução à Comutação IP por Rótulos Através de MPLS. In: Simpósio Brasileiro de Redes de Computadores, 19., 2001, Florianópolis. Anais... Florianópolis: UFSC, 2001.

Nichols, K. et al. (1998) Definition of the Differentiated Services Field (DS field) in the IPv4 and IPv6 Headers. [S.1.]: IETF, Dec. 1998. (RFC 2474).

Li, T.; Rekhter, Y (1998). Provider Architecture for Differentiated Services and Traffic Enginnering (PASTE). [S.1.]: IETF, Oct. 1998. (RFC 2430).

Pieda, P. et al. (2000) A Network Simulator Differentiated Services Implementation. Toronto: Nortel Networks, 2000.

Rajan, R. et al. (1999) A Policy Framework for Integrated and Differentiated Services in the Internet. IEEE Network Magazine, New York, v. 13, n. 5, p. 36-41, 1999.

Rosen E.; Viswanathan A.; Callon R. (2001) Multiprotocol Label Switching Architecture. [S.1.]: IETF, Jan. 2001. (RFC 3031). 
Swallow, G. (1999) MPLS Advantages for Traffic Engineering. IEEE Communications Magazine, New York, v. 37, n. 12, p. 54-57, 1999.

The Network Simulator ns-2. (2002) Disponível em: <http://www.isi.edu/nsnam/ns/index.html. Xiao, X.; Ni, L. (1999) Internet Qos: A Big Picture. IEEE Network Magazine, New York, v. 13, n. 2, p. 8-18, 1999.

Xiao, X.; Hannan, A.; et. al. (2000) Traffic Engineering with MPLS in the Internet. IEEE Network Magazine, New York, v. 14, n. 2, p. 28-33, 2000. 